\title{
New Trends Towards Enhanced Structural Efficiency and Aesthetic Potential in Tall Buildings: The Case of Diagrids
}

\author{
Created by: Domenico Scaramozzino ${ }^{1}$, Alberto Carpinteri ${ }^{2}$, Giuseppe Lacidogna ${ }^{3}$ \\ 1, Department of Structural, Geotechnical and Building Engineering, Politecnico di Torino, \\ Corso Duca degli Abruzzi 24, 10129, Torino, Italy; domenico.scaramozzino@polito.it \\ 2, Politecnico di Torino; alberto.carpinteri@polito.it \\ 3, Department of Structural, Geotechnical and Building Engineering, Politecnico di Torino, \\ 24, Corso Duca degli Abruzzi, 10129 Torino, Italy; giuseppe.lacidogna@polito.it
}

Version received: 15 June 2020

Due to the increasing number of people and activities within the cities, tall buildings are exploited worldwide to address the need for new living and commercial spaces, while limiting the amount of used land. In recent decades, the design of tall buildings has undergone a remarkable improvement, thanks to the development of new computational tools and technological solutions. This has led to the realization of innovative structural systems, like diagrids, which allow the achievement of high structural performances and remarkable architectural effects. In this contribution, a thorough and updated review of diagrid structural systems is provided. Simplified methodologies for the preliminary design and structural analysis are reported. Special attention is also paid to the optimization of the structural response based on the geometrical pattern. A discussion of the effect of local deformability, stability and shear-lag phenomenon is carried out. Results from nonlinear and dynamic analyses for the seismic assessment of diagrid systems are reported, and attention is also paid to the recent research on diagrid nodes. Eventually, an overview of twisted, tapered, tilted and freeform diagrid towers is carried out, with a final mention of hexagrids, another recent evolution of tubular systems for tall buildings.

The evolution of tall buildings has undergone remarkable developments in the last century. The first buildings reaching a few tens of stories were first built in the United States in the late 19th century, mostly in the cities of New York and Chicago. At the beginning of the 20th century, a race for the realization of the tallest skyscrapers took place, which led to the completion of the 102-story tall Empire State Building in 1931 (Figure 1a). Although at that time the height of those buildings was worthy of note, their realization was not achieved by means of significant technological innovations. They usually employed the same steel frames which were adopted for shorter buildings, leading to excessive material usage and rather over-designed solutions ${ }^{[1]}$. Bracings were employed to withstand lateral loads arising from wind pressures and earthquake actions. It was already recognized that lateral actions usually govern the design solutions in tall buildings. In fact, as the building becomes taller, the lateral drifts turn out to be more critical, and there is greater demand for suitable structural systems to carry lateral forces. This leads to a dramatic increase of material consumption with the increase in the number of stories, which is usually referred to as the "premium for height"[1][2].

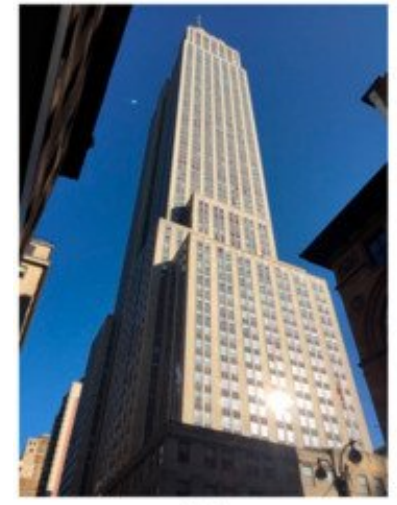

(a)

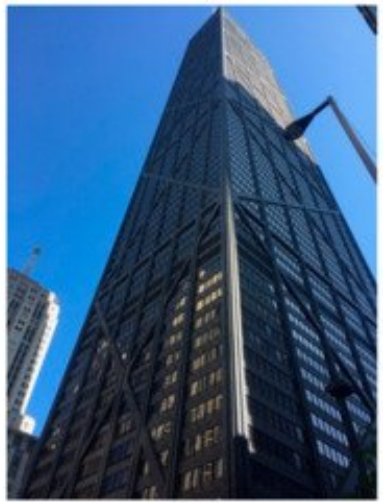

(b)

Figure 1. Different structural systems for 20th century tall buildings: ( a) Moment resisting frame: Empire State Building (New York, NY, USA); ( b) Braced tube: John Hancock Center (Chicago, IL, USA). Pictures taken by D. Scaramozzino.

Due to aesthetic and constructability considerations, the bracings were usually embedded within the interior core of the building. Although their shear resistance, based on the axial deformation of the diagonals, was beneficial in resisting the lateral actions, compared to the mechanism of the conventional moment resisting frames, their placement within the interior of the building prevented their effective employment in withstanding the overturning moment. Therefore, new solutions exploiting bracings on the external perimeter of the building were developed. One of the first examples was the 100-story tall John Hancock Center built in Chicago in 1970 (Figure 1b). The John Hancock is an example of braced tube, where the mega-diagonals spanning over several stories are effective in resisting the shear and bending moment deriving from lateral actions. The braced 
tube was a variation of the typical framed tube, where closely spaced perimeter columns were in charge of providing the necessary lateral stiffness. The adoption of mega-diagonals on the external surface offered higher lateral stiffness, while reducing some detrimental phenomena of the framed tube, such as the shear-lag effect. With this new solution, a greater number of stories and an overall enhanced structural performance could be achieved, leading also to important advantages from a material consumption perspective.

Based on the structural behavior of the braced tube, where vertical columns and external bracings were designed to carry gravity and lateral loads respectively, it was realized that the external mega-diagonals were able to resist vertical and horizontal loads simultaneously, without the need for conventional vertical columns. This led to the realization of the diagrid ("diagonal" + "grid") structural system. The idea of removing vertical columns and considering only inclined diagonals was not new. As a matter of fact, the first diagrid structure was realized before the construction of the John Hancock braced tube, in the 1920s, by the Russian architect Vladimir Shukhov, for the realization of a broadcasting tower in Moscow[3]. The external pattern, made up of a triangular tessellation, allowed the reduction of the wind load while reaching a stable stiff configuration. The first application of a diagrid system in building design occurred in the 1960s, with the completion of the 13-story tall IBM Building (Pittsburgh, USA). The steel diagrid exoskeleton was integrated with the glazing system, and assisted in the overall stability of the building $[3]$.

However, it was not until the early 21 st century that diagrid systems started to be thoroughly applied in the design and construction of tall buildings. The first examples are the Hearst Tower in New York (Figure 2a) and the 30 St. Mary Axe (also known as Swiss Re Tower or The Gherkin) in London (Figure $2 \mathrm{~b}$ ), both by Sir Norman Foster. These buildings reached $180 \mathrm{~m}$ and provided the first references for the suitability of diagrid systems in tall building design. Thanks to the stiff diagrid façades, which create a pleasant diamond-like pattern, the Hearst Tower was realized using $20 \%$ less steel than an equivalent conventional moment frame structure. The aerodynamic form of the Swiss Re Tower, obtained through an external free-form diagrid envelope, allowed the reduction of wind action on the building, and led to column-free flexible internal spaces. These two examples already showed the valuable features of diagrids for tall buildings: enhanced structural performance, saving of material consumption compared to traditional solutions, and significant aesthetic potential. Many diagrid structures were realized worldwide in the following years, where various forms and shapes were adopted for the external diagrid façades. Among others, examples worthy of note are the Guangzhou Financial Center, the CCTV Tower and the Poly International Plaza in China, the Tornado Tower (Figure 2c) in Qatar, the Capital Gate in Arab Emirates, and the Bow Tower in Canada $[3]$. Nowadays, most of the built diagrid structures are made of steel, mostly due to the easier and faster construction, simpler joints and less expensive formworks ${ }^{[3]}$. However, concrete and composite diagrids are also experiencing an increasing popularity, as they allow the realization of even more complex-shaped diagrid patterns, e.g., the O-14 Building in Dubai.

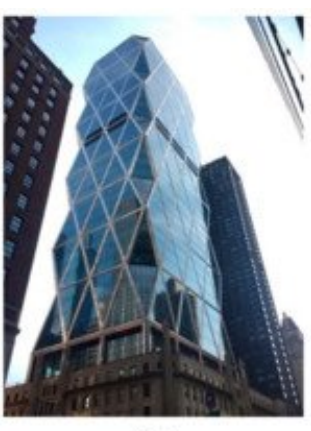

(a)

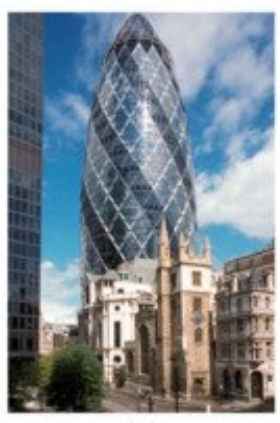

(b)

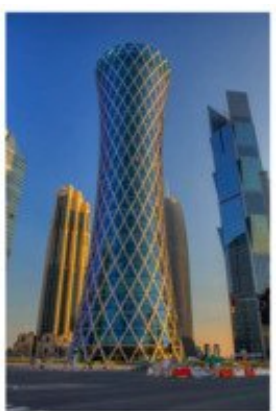

(c)

Figure 2. Examples of diagrid systems in tall buildings: ( a) Hearst Tower (New York, USA), picture taken by D. Scaramozzino; ( b) Swiss Re Tower (London, UK), from https://larryspeck.com/; (c) Tornado Tower (Doha, Qatar), from http://www.asergeev.com/.

The significant use of diagrid systems in recent tall buildings is mainly due to the following reasons: high lateral stiffness (thus low lateral deformability), which allowed the reaching of the lateral deflection limit target using a lower amount of structural material compared to other conventional systems; architectural flexibility, allowing a more rational use of the interior space with fewer columns; modularity, which led to the realization of complex-shaped structures of various forms. These three points arise from the successful use of the triangular module coupled with the inherent structural performance of the tubular structure[4][5]. The triangular element, which is made up of two inclined diagonals and a ring beam, is the basic component of the diagrid façade. The diagonals carry the vertical and lateral loads mostly by axial forces (compression or tension). For this reason, they are usually pinned at the panel nodes, as reflected in Figure 3a. Since the inclined diagonals often extend over multiple stories, the external floor beams of intermediate stories are often supported by the diagonals, which consequently induces slight shear and bending stresses on them. However, in preliminary design stages, these are usually neglected when compared to the high axial stresses arising from the vertical and lateral loads on the building. Note that in Figure $3 \mathrm{a}$, a 3-story module is reported, as the height of each diagrid module has the same height as a triangular element. In other research works, as will be shown in further figures in the remainder of the paper, the diagrid module is defined in such a way that it covers two triangular elements. 


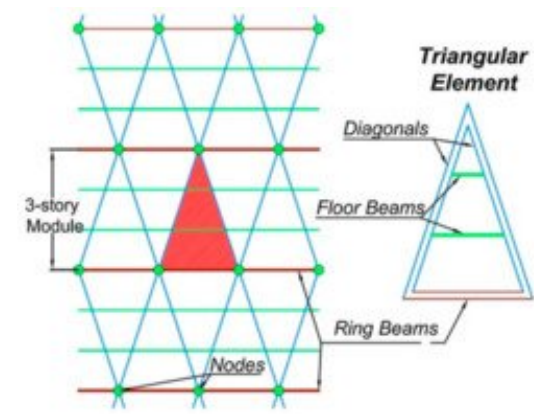

(a)

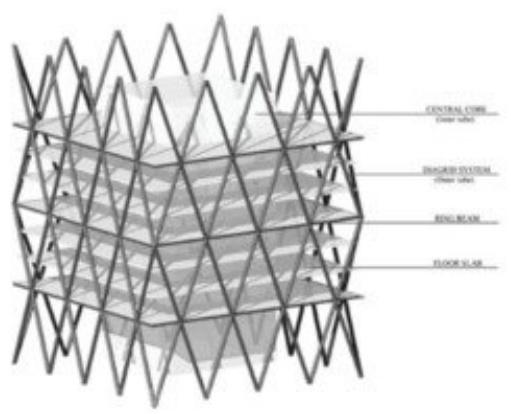

(b)

Figure 3. Fundamental diagrid geometrical features: (a) diagrid module and basic triangular element, used with permission from Asadi and Adeli [5]; (b) diagrid tubular configuration, used with permission from Angelucci and Mollaioli [6].

In Figure $3 \mathrm{~b}$, the three-dimensional view of the tubular diagrid structure is shown, as reported in [6]. Usually, the tube-in-tube configuration is found in real diagrid buildings, where an internal (concrete or steel braced) core is coupled with the external diagrid tube. In preliminary design stages, the diagrid is usually designed to carry the lateral actions alone, while the internal core is designed only for gravity loads. However, further details of the diagrid-core interaction will be shown in the remainder of the paper. Hence, it is the combination of the axial resisting mechanism of the triangular element—characterized by modularity and arrangement flexibility—coupled with the structural efficiency of the tubular configuration, that has ultimately led to the success of the diagrids in recent times.

In this contribution, a thorough and up-to-date survey of the research studies on diagrid systems is reported. In particular, the fundamental stiffness-based approach of the preliminary design is described, as firstly proposed by Moon et al.[7] and further developed in the following years. Moreover, strength-based design methodologies are also discussed, and their implication for the preliminary design is analyzed. The various methodologies available today, besides the typical Finite Element Method (FEM), are also described for the structural analysis of diagrid structures in preliminary design stages, e.g., hand-based calculations[], modular[9] and matrix-based[10] methods, etc. Furthermore, the subject related to the optimization of the diagrid performance based on its geometrical features is also addressed. This problem has been thoroughly tackled by various researchers in the last decade, with different methodologies[11][12], and has led to significant results. The problem of local structural issues in the design of diagrid tall buildings is also addressed, e.g., excessive inter-story drifts and stability of interior columns. The mathematical formulation for identifying these problems is reported, as well as the solutions which have been suggested to tackle them, such as the insertion of secondary bracing systems (SBSs), as was first proposed by Montuori et al[13]. The shear-lag phenomenon in diagrid tubes is discussed and its influence, depending on the diagrid's geometrical parameters, is analyzed. The research studies which have dealt with the nonlinear behavior of diagrid tubes, in order to assess their seismic and robustness performance, are also discussed. The recent research on diagrid nodes, which represents a crucial component in the correct behavior of the diagrid, is also reported. Comments are also provided about the new trends regarding unconventional diagrids, which are applied for the realization of twisted, tilted, tapered and freeform buildings. A further evolution of the grid tubular structure, which has experienced a significant growth in recent tall building design, is finally presented.

\section{References}

1. Mir M. Ali; Kyoung Sun Moon; Structural Developments in Tall Buildings: Current Trends and Future Prospects. Architectural Science Review 2007, 50, 205-223, 10.3763/asre.2007.5027.

2. Mir M. Ali; Kyoung Sun Moon; Advances in Structural Systems for Tall Buildings: Emerging Developments for Contemporary Urban Giants. Buildings 2018, 8, 104, 10.3390/buildings8080104.

3. Mohammad Reza Maqhareh; Sepideh S. Korsavi; The Evolutionary Process of Diagrid Structure Towards Architectural, Structural and Sustainability Concepts: Reviewing Case Studies. Journal of Architectural Engineering Technology 2014, 3, 121, 10.4172/21689717.1000121.

4. Boake, T.M.. Diagrid Structures: Systems, Connections, Details; De Gruyter, Ed.: Basel, Switzerland, 2014; pp. - .

5. Esmaeel Asadi; Hojjat Adeli; Diagrid: An innovative, sustainable, and efficient structural system. The Structural Design of Tall and Special Buildings 2017, 26, e1358, 10.1002/tal.1358.

6. Giulia Angelucci; Fabrizio Mollaioli; Diagrid structural systems for tall buildings: Changing pattern configuration through topological assessments. The Structural Design of Tall and Special Buildings 2017, 26, e1396, 10.1002/tal.1396.

7. Kyoung-Sun Moon; Jerome J. Connor; John E. Fernández; Diagrid structural systems for tall buildings: characteristics and methodology for preliminary design. The Structural Design of Tall and Special Buildings 2007, 16, 205-230, 10.1002/tal.311.

8. Elena Mele; Maurizio Toreno; Giuseppe Brandonisio; Antonello De Luca; Diagrid structures for tall buildings: case studies and design considerations. The Structural Design of Tall and Special Buildings 2012, 23, 124-145, 10.1002/tal.1029.

9. Chengqing Liu; Kaiqiang Ma; Calculation model of the lateral stiffness of high-rise diagrid tube structures based on the modular method. The Structural Design of Tall and Special Buildings 2016, 26, e1333, 10.1002/tal.1333.

10. Giuseppe Lacidogna; Domenico Scaramozzino; Alberto Carpinteri; A matrix-based method for the structural analysis of diagrid systems. Engineering Structures 2019, 193, 340-352, 10.1016/j.engstruct.2019.05.046.

11. Valentina Tomei; Maura Imbimbo; Elena Mele; Optimization of structural patterns for tall buildings: The case of diagrid. Engineering Structures 2018, 171, 280-297, 10.1016/j.engstruct.2018.05.043.

12. Giuseppe Lacidogna; Domenico Scaramozzino; A. Carpinteri; Influence of the geometrical shape on the structural behavior of diagrid tall buildings under lateral and torque actions. Developments in the Built Environment 2020, 2, 100009, 10.1016/j.dibe.2020.100009. 
13. Giovanni Maria Montuori; Elena Mele; Giuseppe Brandonisio; Antonello De Luca; Secondary bracing systems for diagrid structures in tall buildings. Engineering Structures 2014, 75, 477-488, 10.1016/j.engstruct.2014.06.011.

\section{Keywords}

tall buildings; diagrid; preliminary design; structural analysis; stiffness-based methodology; optimization; hexagrid

c) (i) (C) 2020 by the author(s). Distribute under a Creative Commans CC BY license 